\title{
The tragedy of the commons in microbial populations: insights from theoretical, comparative and experimental studies
}

\author{
RC MacLean ${ }^{1,2}$ \\ ${ }^{1}$ NERC Centre for Population Biology, Imperial College London, Ascot, UK and ${ }^{2}$ Department of Zoology, University of Oxford, \\ Oxford, UK
}

\begin{abstract}
First principles of thermodynamics imply that metabolic pathways are faced with a trade-off between the rate and yield of ATP production. Simple evolutionary models argue that this trade-off generates a fundamental social conflict in microbial populations: average fitness in a population is highest if all individuals exploit common resources efficiently, but individual reproductive rate is maximized by consuming common resources at the highest possible rate, a scenario known as the tragedy of the commons. In this paper, I review studies that have addressed two key questions: What is the evidence that the rate-yield trade-off is an evolutionary constraint on metabolic pathways? And, if so, what determines evolutionary outcome of the conflicts generated by this trade-off? Comparative studies and microbial experiments provide evidence that the rate-yield trade-off is an evolutionary constraint that is driven by thermodynamic constraints that are common to all metabolic pathways and
\end{abstract}

pathway-specific constraints that reflect the evolutionary history of populations. Microbial selection experiments show that the evolutionary consequences of this trade-off depend on both kin selection and biochemical constraints. In wellmixed populations with low relatedness, genotypes with rapid and efficient metabolism can coexist as a result of negative frequency-dependent selection generated by density-dependent biochemical costs of rapid metabolism. Kin selection can promote the maintenance of efficient metabolism in structured populations with high relatedness by ensuring that genotypes with efficient metabolic pathways gain an indirect fitness benefit from their competitive restraint. I conclude by suggesting avenues for future research and by discussing the broader implications of this work for microbial social evolution.

Heredity (2008) 100, 233-239; doi:10.1038/sj.hdy.6801073; published online 24 October 2007

Keywords: experimental evolution; kin selection; tragedy of the commons; cooperation; trade-off; spatial structure

\section{Introduction}

Whenever individuals have access to a common resource that can be exploited either rapidly (units energy produced/units time) or efficiently (units energy produced/units resource consumed) an evolutionary dilemma is created: average fitness in a population is highest if all individuals exploit common resources efficiently, but individual reproductive rate is maximized by consuming common resources at the highest possible rate. In an influential paper, Hardin (1968) argued that this conflict selects for increasingly selfish resource use, because the benefit an individual gains from increasing its resource exploitation rate is much less than the cost it pays in terms of reduced available resources, a scenario that he termed the 'tragedy of the commons'. For example, consider the case of competition between two different resource exploitation strategies: a prudent strategy that consumes resource at a rate 1 resource/time with a yield of 1 energy/resource, and selfish strategy that consumes resources at a rate $(1+s)$ resource/time with a yield of $(1-s)$ energy/resource. The benefit of acting selfishly in

Correspondence: Dr RC MacLean, Department of Zoology, University of Oxford, South Parks Road, Oxford OX1 3PS, UK.

E-mail: craig.maclean@zoo.ox.ac.uk

Received 9 May 2007; accepted 22 September 2007; published online 24 October 2007 this case is equal to $s$, but the cost of this strategy, in terms of reduced resources for future reproduction, is $-s / N$, where $N$ is population size, because all individuals in the population have access to the same pool of resources. The logic of the tragedy of the commons predicts that individual good will be maximized with disastrous consequences for the population. In an extreme scenario, this social conflict between the interest of individuals and the group could conceivably result in extinction of the population. What prevents this outcome from being realized?

In order for resource competition to generate social conflict, it is clear that there must be a trade-off between the benefit that an individual gains from resource exploitation and the benefit that the group gains. Although there is some evidence for such trade-offs in multicellular eukaryotes (for example, Mueller et al., 1991), no general explanations (that I know of) have been offered to explain why such trade-offs must exist. In the case of microorganisms, it is well-established that a trade-off between the rate and yield of metabolism is a fundamental aspect of resource use, making microbes an ideal group of organisms in which to investigate the social conflicts created by resource competition. In this paper, I review theoretical studies relating to resource competition and social conflict in microbial populations and experimental tests of this theory using experimental 
evolution in microbial microcosms. I then synthesize the conclusions of this literature, suggest possible avenues for future research, and discuss the relevance of this work for our understanding of social evolution.

\section{Metabolic constraints generate social conflicts}

Heterotrophic microorganisms generate the energy required for growth and reproduction by using metabolic pathways to convert extracellular resources with high free energy into products with low free energy, conserving some of the energy difference in the form of ATP. In the case of ATP-producing reactions, the existence of a trade-off between the rate and yield of energy production can be mathematically derived from first principles of thermodynamics (Heinrich et al., 1997; Waddell et al., 1997; Pfeiffer et al., 2001). The logic of this trade-off can be illustrated simply (Figure 1a). Consider the example of a catabolic reaction that converts a substrate, $S$, and ADP into a product, $P$, and ATP. Thermodynamics tells us that rate of a reaction is linearly related to the difference in free energy between substrates and products (the Gibbs free energy change). If all of the difference in free energy between $S$ and $P$ is conserved in the form of ATP (yield =1), there will be no difference in free energy between substrates and products and the reaction cannot be driven forward. At the other extreme, consider a reaction in which almost none of the free energy difference between substrate and product is conserved in the form of ATP (yield $<1$ ). The free energy of the products $(P+$ ATP $)$ is less than that of the substrates, allowing this reaction to proceed at a rate $>0$ and at a yield of $<1$.

Metabolic pathways, of course, are made up of many reactions, only some of which produce ATP. Are tradeoffs between rate and yield still present at the level of entire pathways? The simplest method to test for a rateyield trade-off is to measure the yield of microbial cultures grown at different resource uptake rates. Such experiments consistently find evidence of a trade-off between the rate and yield of entire metabolic pathways (Weusthuis et al., 1994; Kreft, 2004; Gudelj et al., 2007). For example, a very convincing trade-off between the rate and yield of glucose metabolism has been demonstrated in the yeast Saccharomyces cerevisiae (Figure 1b). Evidence that the rate versus yield trade-off acts as an evolutionary a

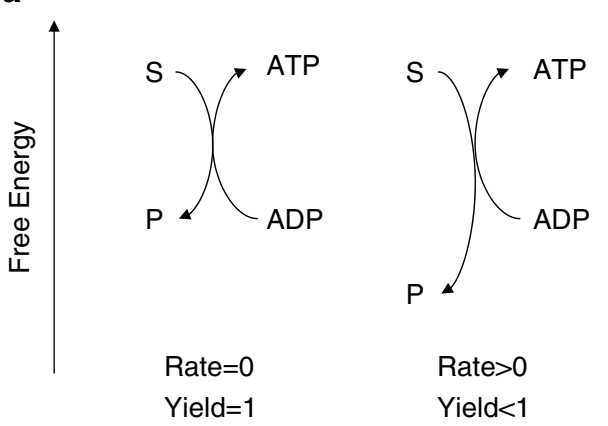

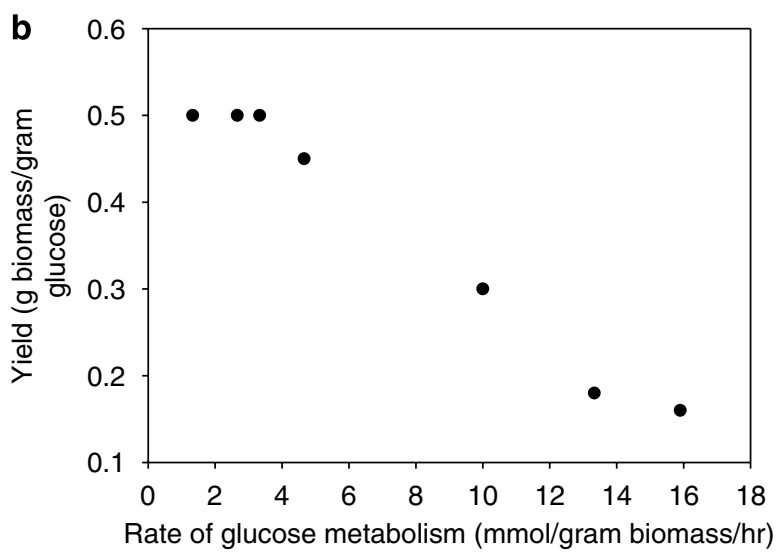

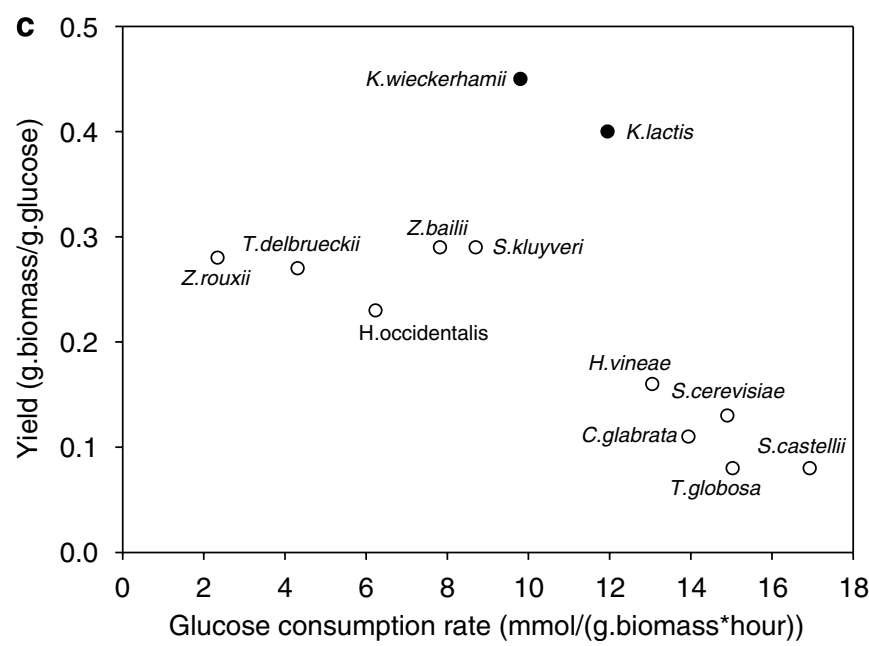

Figure 1 The rate-yield trade-off. Panel a shows a schematic of the thermodynamic trade-off between the rate and yield of ATP production in an individual catabolic reaction. Plotted points in panel $\mathbf{b}$ show the rate and yield of glucose metabolism in glucose-limited chemostat cultures of Saccharomyces cerevisiae. Data taken from Postma et al. (1989). Plotted points in panel c show the rate and yield of glucose metabolism in exponentially growing cultures of yeast species. Data are taken from Merico et al. (2007). Species that ferment glucose to produce ethanol are shown with open symbols and those species that do not produce ethanol are shown with filled symbols. The negative correlation between the rate and yield of metabolism is significantly different from zero after correcting for phylogeny, as revealed by comparing the sign of the correlation between the rate and yield of metabolism between sister taxa $(P=0.03)$. 
constraint, and not just a physiological constraint, comes from the observation that the rate and yield of glucose metabolism are negatively correlated among species of yeasts (Figure 1c).

\section{Theoretical models}

What are the evolutionary consequences of the metabolic rate-yield trade-off? Early theoretical studies addressed this question by modeling pairwise competition between hypothetical strains of microbe that consume resources rapidly or efficiently. The outcome of these models is simple: under competition for a common resource, the selfish strain with a high rate of ATP production always displaces the prudent strain with a high yield of ATP production, revealing the potential for metabolic trade-offs to generate a tragedy of the commons (Pfeiffer et al., 2001; Frick and Schuster, 2003; Kreft, 2004; Gudelj et al., 2007). This outcome changes when individuals compete for local pools of resources with their neighbors, as occurs in structured populations such as a bacterial biofilm (Kreft, 2004). In this case, strains with a high yield of ATP metabolism can displace competitors with a high rate of ATP metabolism by effectively exploiting their local pool of resources (Pfeiffer et al., 2001; Kreft, 2004). In this scenario, rapid metabolism is still associated with a fitness benefit, because it increases reproductive rate, but the cost of rapid ATP production is not borne by the population as a whole. Instead, the cost of rapid ATP production is paid exclusively by local groups of cells with rapid metabolism, which leads to an indirect cost of selfishness. Although theoretical models of resource competition and social conflict in microorganisms differ with respect to their ecological and biochemical assumptions, they lead to the common prediction spatially structured ecological interactions are the key to preventing the tragedy of the commons in microbial populations. These theoretical results are consistent with kin selection theory (Hamilton, 1963, 1964). According to Hamilton's rule, a gene for altruism can only spread by natural selection when $r b>c$, where $r$ is the relatedness between interacting individuals, $b$ is the benefit to the recipient of the altruistic act and $c$ is the cost to the actor. In structured populations, interactions are strongest between neighbors, who are likely to be kin (that is, clone mates), and relatedness is high, generating strong kin selection for altruism. In mixed populations, interactions between individuals are random, which results in low relatedness and weak selection for altruism.

\section{Experimental evolution}

To test the predictions of these models MacLean and Gudelj (2006) developed an experimental system based on competition between two strains of yeast with alternative pathways of glucose metabolism (Otterstedt et al., 2004): a prudent respirer strain (R) and a selfish respiro-fermenter strain (R-F). In $S$. cerevisiae, glucose metabolism is controlled by the rate of glucose flux into the cell (Elbing et al., 2004). At low glucose flux, yeast completely oxidizes all of the glucose it ingests by the tricarboxylic acid (TCA) cycle, resulting the production of approximately 32 molecules of ATP per molecule of glucose ingested. As the rate of glucose flux into the cell increases, the TCA cycle becomes saturated and excess glucose is fermented by glycolysis, resulting in the production of only two ATP per molecule of fermented glucose. The $\mathrm{R}$ strain of $S$. cerevisiae used by MacLean and Gudelj (2006) has a maximal glucose uptake rate equal to the maximal rate of the TCA cycle, ensuring that this strain completely oxidizes all of the glucose it ingests. The selfish R-F strain, on the other hand, has a maximal glucose uptake rate $\approx 10$ times the maximal rate of the TCA cycle so that this strain is able to use fermentation to produce ATP at a higher rate and at a lower yield than the obligate respirer strain (Otterstedt et al., 2004).

Competition experiments between $\mathrm{R}$ and R-F produced a surprising result: both strains were able to coexist in a seasonal environment with low relatedness (batch cultures) as a result of negative frequencydependent selection, demonstrating that kin selection is not necessary to avoid the tragedy of the commons (Figure 2a). Mathematical models of competition and subsequent experiments established that coexistence is attributable to the effects of metabolic by-products of fermentation. When yeast ferments glucose, it produces not only ATP, but also toxic metabolic by-products (predominantly ethanol, but also small quantities of acetate) that passively diffuse out of the cell. The production of toxic metabolites is deleterious to the population as a whole, but the cost of toxin production is primarily borne by fermenting cells that act as a source of toxins, simply because these molecules are transported by diffusion (see MacLean and Gudelj, 2006 for mathematical support for this argument). Interestingly, this cost of selfish metabolism depends on the density of fermenting cells: when the density of fermenting cells is low, the total concentration of toxins in the environment is low and a steep diffusion gradient allows fermenting cells to rid themselves of toxins. At high fermenter density, toxins rapidly accumulate in the environment, making it difficult for fermenting cells to rid themselves of the toxins they produce as a direct consequence of selfish metabolism. It is this density-dependent cost of selfish metabolism that generates negative frequencydependent selection that maintains the coexistence of cells with rapid and efficient glucose metabolism. Although this cost of selfish metabolism does not reflect a thermodynamic trade-off between the rate and yield of ATP metabolism, the net effect is the same: cells with a high rate of ATP production are punished in terms of a low reproductive yield (biomass produced/ATP produced) because of the toxic effects of metabolites that are formed as a direct and necessary consequence of rapid ATP production.

To investigate the role of kin selection, the R and R-F strains were competed against each other in a metapopulation made up of 96 discrete patches provided by a microtiter plate. In this experiment, population structure generates high relatedness, which should promote cooperation. Populations from all patches were then pooled to determine the outcome of competition at the level of the metapopulation. In this experiment, fitness was positive frequency-dependent, implying that either metabolic strategy can resist invasion by the alternative strategy (Figure $2 \mathrm{~b}$ ). This result can be explained simply. The respirer strain can benefit from its high yield of ATP production due to kin selection when it escapes direct 

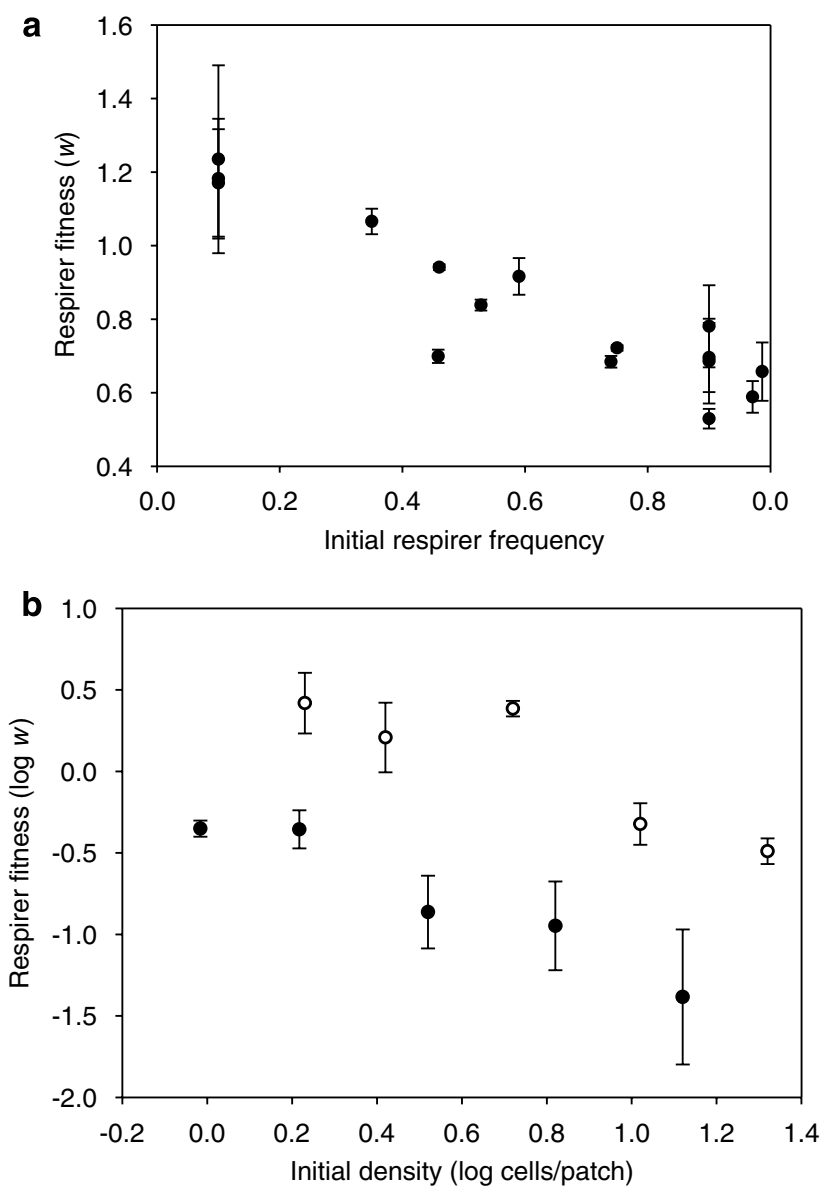

Figure 2 Competition between strains of $S$. cerevisiae with a high rate and high yield of metabolism. This figure shows the outcome of competition between isogenic respirer and respiro-fermenter strains of yeast in well-mixed (a) and structured (b) microcosms. Fitness was measured as $w$, the ratio of doublings of respirers relative to respiro-fermenters during competition, such that a value of $w=1$ represents equal competitive fitness. Error bars show the standard error associated with each fitness estimate. In (b) the fitness of the respirer was measured in metapopulations that were initially dominated by respirers (open symbols) and respire-ferments (closed symbols). Reproduced from MacLean and Gudelj (2006) with kind permission of the authors and Nature Publishing Group.

competition from R-F by colonizing resource patches that lack R-F (that is, at high relatedness), as is expected to occur when the frequency of the respirer strain is high, or the overall density of the population is low. Collectively, these experiments provide an important demonstration that both kin selection and biochemical constraints (the rate-yield trade-off) determine the outcome of social conflicts generated by resource competition.

An important limitation of this study is that it considered only short-term competition between two variants with alternative metabolic pathways. What is the outcome of this conflict likely to be over long time scales, when de novo mutation allows populations to explore a continuous range of metabolic strategies? Novak et al. (2006) assayed the rate and yield of glucose metabolism in populations of $E$. coli B that were propagated in a spatially homogeneous glucose-limited environment under strong selection for increased growth rate (Vasi et al., 1994) for 20000 generations. Initially, populations increased both the rate and yield of glucose metabolism and, after 20000 generations of selection, no evidence of a rate-yield trade-off could be found by comparing the mean growth rate and yield of 12 replicate populations (Figure 3a and b). However, assays of clones isolated from 4 focal populations after 20000 generations of selection revealed conclusive evidence of a trade-off between the rate and yield of growth in all 4 populations (Figure 3c).

Novak et al. (2006) explain these results as follows. The ancestor that was used to start this experiment was poorly adapted to its environment and was able to increase both its growth rate and yield because it had never been strongly selected for either rate or yield in its recent evolutionary past. Populations diverged during the course of experiment, because chance and epistasis resulted in the fixation of different beneficial mutations in different replicate populations (Travisano et al., 1995). The negative genetic correlation between rate and yield within populations provides evidence of the existence of a trade-off between the rate and yield of ATP production, but the lack of a negative correlation between populations suggests that this constraint was in part attributable to mechanistic constraints imposed by the different adaptations to glucose limitation in different populations. In other words, although all 12 of these populations faced the same underlying thermodynamic constraints on the rate and yield of ATP production, the actual trade-offs that emerged were dictated by both a common thermodynamic constraint and by a population-specific constraint that emerged as a consequence of the unique evolutionary history of each population. This is an intriguing hypothesis that deserves further experimental tests. For example, it would be possible to measure the rate-yield trade-off in engineered strains of E. coli carrying known combinations of the mutations that are known to have contributed to adaptation in the long-term E. coli evolution experiment. The comparative biochemistry of yeasts provides evidence for the kind of population-specific constraints invoked by Novak et al. (2006): although the rate-yield trade-off appears to be a universal constraint on the glucose metabolism of yeasts, the cost of a high rate of metabolism is much higher in species that ferment glucose to produce ethanol relative to species that form less toxic metabolic by-products (Figure 1b).

An interesting finding of this study is that withinpopulation genetic variation in rate and yield can be maintained over long time scales (Figure 3c). How can we explain the maintenance of this diversity? Competition experiments in yeast suggest that costs associated with a high rate of ATP production might generate negative frequency-dependent selection that actively maintains this variation. In support of this argument, it has been shown that negative frequency-dependent maintains genetic variation in these evolving populations of E. coli (Elena and Lenski, 1997; Rozen and Lenski, 2000), but the critical experiments to test the role of metabolite toxicity in generating negative frequencydependent selection have not been carried out, and it is clear that alternative mechanisms, including crossfeeding of metabolites and trade-offs between glucose uptake rate and affinity, contribute to negative frequencydependent selection in at least one of the populations (Rozen and Lenski, 2000). 
a
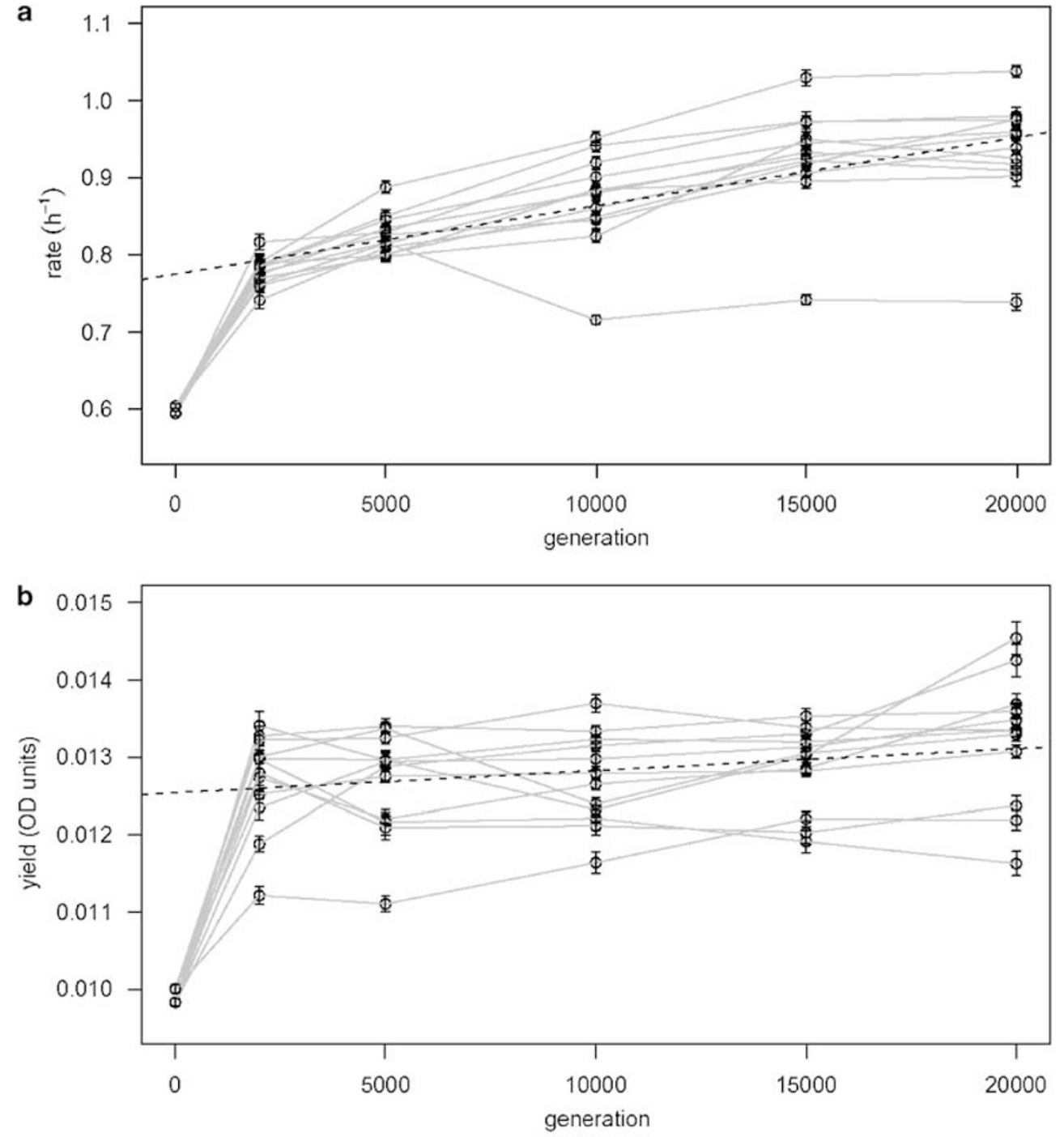

C

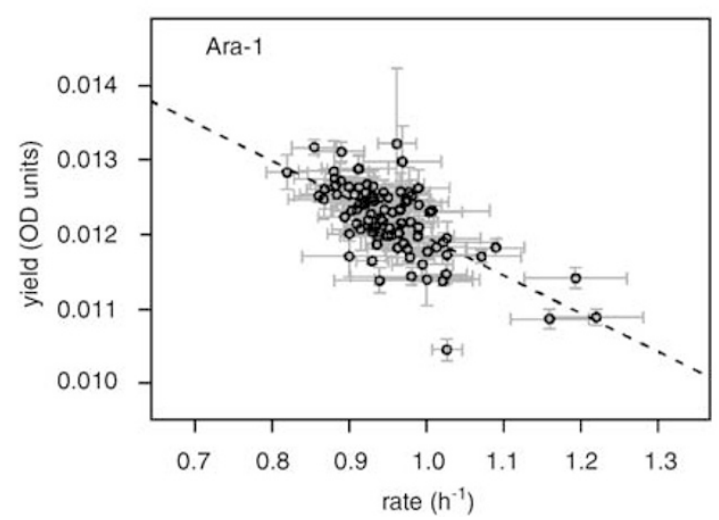

Figure 3 Long-term evolution of rate and yield in experimental populations of E. coli. Plotted points show the rate (panel a) and yield (panel b) of glucose metabolism in 12 populations of $E$. coli that were selected in glucose-limited batch cultures over a period of 20000 generations. In (a) and (b) dashed lines show the regression of rate or yield against time, after accounting for the rapid initial increase in both rate and yield in the first 1000 generations of selection and error bars represent standard errors. Plotted points in panel $\mathrm{c}$ show the rate and yield ( \pm s.e.) of individual clones sampled from one population (Ara -1) after 20000 generations of selection and the dashed line shows a linear regression of rate against yield. Similar results were found in other populations. Reproduced from Novak et al. (2006) with kind permission of the authors and the University of Chicago Press.

\section{Conclusion}

Resource competition and social conflict in microbial populations is a subject that has attracted growing interest since the publication of a landmark paper by Pfeiffer et al. (2001). Empirical evidence from a number of systems shows that the rate-yield trade-off imposes an evolutionary constraint on metabolic pathways, and it is 
clear that the actual trade-offs that emerge are driven by both underlying thermodynamic constraints and by mechanistic constraints that reflect the unique evolutionary history of populations. Experimental studies have demonstrated that the evolutionary consequences of this trade-off are determined by both kin selection and biochemical constraints. Despite the generality of these conclusions, it is important to emphasize that they are based on a small number of studies, and it might be possible to make considerable progress in this field by increasing the ecological and biochemical breadth of studies examining resource competition and social conflict in microbes.

Early theoretical studies that used a variety of modeling approaches consistently concluded that the rate of ATP production should evolve to its maximal possible value in a homogeneous environment and, as a consequence, the yield of ATP production should evolve to a minimal possible value. Experimental studies in both $S$. cerevisiae and E. coli do not support this prediction: negative frequency-dependent selection can maintain the coexistence of strains with a high rate and high yield of metabolism, even in simple environments that lack spatial heterogeneity. In the case of yeast, it is known that negative frequency-dependent selection stems from the density-dependent toxic effects of metabolites produced as a direct consequence of rapid ATP production. How general is this phenomenon? Although the production of excess metabolites during rapid growth (that is fermentation) is a feature of the biochemistry of many microorganisms, it is clear that the toxic effects of rapid metabolism depend in part on the pathway under consideration. For example, species of yeast that do not form ethanol as the primary by-product of fermentation have much higher yields of glucose metabolism than species that produce this toxic metabolite (Figure 1c). Thermodynamic models also predict that in some cases metabolic pathways with the same substrates and products can nonetheless operate at high rate or high yield (Aledo and del Valle, 2004). These biochemical observations suggest that negative frequency-dependent selection due to metabolite toxicity many not be a universal explanation for the maintenance of efficient metabolism.

The second key prediction of early theoretical models is that population structure promotes the maintenance of a high yield of ATP production by kin selection. The only test of this hypothesis has provided good evidence that kin selection in structured populations generates positive frequency-dependent selection that can maintain a high yield of ATP production (MacLean and Gudelj, 2006). Further support for this hypothesis comes from a recent selection experiment with lytic phage (Kerr et al., 2006). Two key parameters in the life history of phage are the latent period, the time interval between the infection of a bacterial cell and lysis of the host cell, and burst size, the number of progeny released at lysis. From the perspective of phage, latent period is equivalent to the rate of resource exploitation and burst size is equivalent to the yield of resource exploitation. Kerr et al. (2006) selected phage in metapopulations containing a host, E. coli that acts as a resource. In order to investigate the role of kin selection, they manipulated the rate of migration between patches of bacteria and phage. At high dispersal rate (low relatedness), phage with a high resource consumption rate (short latent period) and low yield (low burst size) evolved, whereas at low dispersal rate (high relatedness), phage with a long latent period and high burst size evolved.

\section{Phenotypic plasticity}

For the purpose of simplicity, it has been assumed throughout this review that genotypes have a fixed rate and yield of metabolism. It is clear, however, that an optimal strategy may be to alter the rate and yield and metabolism depending on prevailing abiotic conditions. Does such plasticity in metabolic strategy exist? Using thermodynamic models, Aledo and del Valle (2004) have argued that as extracellular glucose availability and, therefore, glycolytic flux increases, yeast glycolysis shifts from a strategy of efficient energy production to a strategy of rapid energy production by coupling the rate of ATP production by glycolysis to the rate of ATP consumption by anabolic reactions. These arguments are compelling, but they could benefit from selecting yeast and high, low, and variable glucose availability, with the expectation that increased plasticity will only evolve in a fluctuating environment.

\section{Competitive restraint as a form of cooperation}

Evolutionary biologists typically define cooperative social strategies as traits that are costly to individuals, but beneficial to the group. Numerous examples of cooperation can be found in microbes (reviewed in Crespi, 2001) and in most cases, this involves direct investment by secreting costly macromolecules such as digestive enzymes and siderophores that produce a public good that benefits the group. The efficient consumption of common resources (competitive restraint) represents a very primitive form of cooperation: by using metabolic pathways with a high yield of ATP production, a microbe sacrifices its current reproductive rate in favor of efficient group reproduction. How are the conditions for the emergence of 'competitive restraint' and direct investment in the common good related? This question has not yet been addressed in either a theoretical or experimental context. In many cases, the extracellular enzymes secreted by 'cooperative' microbes produce common resources that can be exploited rapidly or efficiently. For example, yeast can secrete the enzyme invertase to catalyze the extracellular hydrolysis of sucrose into glucose and fructose. Invertase secretion is clearly a cooperative trait (Greig and Travisano, 2004): the enzyme is costly to produce, but invertase secretion provides a benefit to the group by converting an inaccessible resource into glucose, which can then be metabolized using either rapid or efficiently. How do strategies for the production and consumption of extracellular resources coevolve? Again, this is a question that deserves both theoretical and experimental investigation.

\section{The ecology of social conflict}

Theoretical and experimental studies have shown that ecological interactions can be important determinants of the outcome of social conflict created by the rate-yield trade-off, but the range of ecological scenarios explored 
in these studies has been rather limited. Verbal arguments suggest that simple forms of environmental variation, such as variation in productivity and disturbance rate, could have a profound impact on the evolutionary outcome of the rate-yield trade-off, but it should be relatively straightforward to explicitly investigate these ideas using mathematical and experimental methods that have already been developed in this literature.

\section{Outlook}

The metabolic rate-yield trade-off appears to be a universal feature of metabolic pathways that creates a profound social conflict in microbial populations. Considerable progress toward understanding how this conflict is resolved has been made by developing mathematical models that integrate theoretical aspects of biochemistry with ecological principles and testing these models using microbial selection experiments. Future work in this emerging field should focus on the evolutionary consequences of phenotypic plasticity in metabolic pathways, the co-evolution of different forms of metabolic cooperation, and the role of environmental variation in pathway evolution. Moreover, it would be desirable to integrate the theoretical literature on the rate-yield trade-off with kin selection theory by developing kin selection models that explicitly incorporate the density-dependent costs and benefits of altruism that are such a prominent feature of this social conflict.

\section{Acknowledgements}

This work was funded by grants from NERC (UK) to the Centre for Population Biology. I would like to thank Ivana Gudelj and Thomas Pfeiffer for interesting discussions on this topic and Graham Bell, Stu West and an anonymous reviewer for their comments on an earlier draft of this paper.

\section{References}

Aledo JC, del Valle AE (2004). The ATP paradox is the expression of an economizing fuel mechanism. J Biol Chem 279: 55372-55375.

Crespi BJ (2001). The evolution of social behavior in microorganisms. Trends Ecol Evol 16: 178-183.

Elbing K, Larsson C, Bill RM, Albers E, Snoep JL, Boles E et al. (2004). Role of hexose transport in control of glycolytic flux in Saccharomyces cerevisiae. Appl Env Microbiol 70: 5323-5330.

Elena SF, Lenski RE (1997). Long-term experimental evolution in Escherichia coli 7. Mechanisms maintaining genetic variability within populations. Evolution 51: 1058-1067.

Frick T, Schuster S (2003). An example of the prisoner's dilemma in biochemistry. Naturwissenschaften 90: 327-331.
Greig D, Travisano M (2004). The prisoner's dilemma and polymorphism in yeast SUC genes. Proc Roy Soc Lond B 271: S25-S26.

Gudelj I, Beardmore RE, Arkin SS, Maclean RC (2007). Constraints on microbial metabolism drive evolutionary diversification in homogeneous environments. J Evol Biol 20: $1882-1889$.

Hamilton WD (1963). The evolution of altruistic behavior. Am Nat 97: 354-356.

Hamilton WD (1964). The genetical evolution of social behaviour. I. J Theor Biol 7: 1-16.

Hardin G (1968). The tragedy of the commons. Science 162: 1243-1248.

Heinrich R, Montero F, Klipp E, Waddell T, Melendez-Hevia E (1997). Theoretical approaches to the evolutionary optimization of glycolysis: thermodynamic and kinetic constraints. Eur I Biochem 243: 191-201.

Kerr B, Neuhauser C, Bohannan BJM, Dean AM (2006). Local migration promotes competitive restraint in a host-pathogen 'tragedy of the commons'. Nature 442: 75-78.

Kreft J-U (2004). Biofilms promote altruism. Microbiology 150: 2751-2760.

MacLean RC, Gudelj I (2006). Resource competition and social conflict in experimental populations of yeast. Nature 441 : 498-501.

Merico A, Sulo P, Piskur J, Compagno C (2007). Fermentative lifestyle in yeasts belonging to the Saccharomyces complex. FEBS J 274: 976-989.

Mueller L, Guo P, Ayala F (1991). Density-dependent natural selection and trade-offs in life history traits. Science 253 433-435.

Novak M, Pfeiffer T, Lenski RE, Sauer U, Bonhoeffer S (2006). Experimental Tests for an Evolutionary Trade-Off between Growth Rate and Yield in E. Coli. Am Nat 168: 242-251.

Otterstedt K, Larsson C, Bill RM, Stahlberg A, Boles E, Hohmann S et al. (2004). Switching the mode of metabolism in the yeast Saccharomyces cerevisiae. EMBO Rep 5: 532-537.

Pfeiffer T, Schuster S, Bonhoeffer S (2001). Cooperation and competition in the evolution of ATP-producing pathways. Science 292: 504-507.

Postma EC, Verduyn W, Scheffers WA, van Dijken JP (1989). Enzymatic analysis of the Crabtree effect in glucose-limited chemostat cultures of Saccharomyces cerevisiae. Appl Env Microbiol 55: 468-477.

Rozen DE, Lenski RE (2000). Long-term experimental evolution in Escherichia coli. VIII. Dynamics of a balanced polymorphism. Am Nat 155: 24-35.

Travisano M, Mongold JA, Bennett AF, Lenski RE (1995). Experimental tests of the role of adaptation, chance, and history in evolution. Science 267: 87-90.

Vasi F, Travisano M, Lenski RE (1994). Long-term experimental evolution in Escherichia coli. II. Changes in life-history traits during adaptation to a seasonal environment. Am Nat 144: 432-456.

Waddell T, Repovic P, Melendez-Hevia E, Heinrich R, Montero F (1997). Optimization of glycolysis: a new look at the efficiency of energy coupling. Biochem Educ 25: 204-205.

Weusthuis RA, Pronk JT, van den Broek PJ, van Dijken JP (1994). Chemostat cultivation as a tool for studies on sugar transport in yeasts. Microbiol Mol Biol Rev 58: 616-630. 